\title{
EFECTO DE LA SEMILLA DE LINAZA (Linum Usitatissimum) EN EL CRECIMIENTO DE RATAS WISTAR
}

\section{EFFECT OF FLAXSEED (Linum Usitatissimum) ON THE GROWTH OF WISTAR RATS}

\author{
Kátia Calvi Lenzi de Almeida (1), Flávia Spreafico Fernandes (2), \\ Gilson Teles Boaventura (2), Maria Angélica Guzmán-Silva (1).
}

(1) Laboratório de Patología Experimental, Departamento de Patología, Facultad de Medicina, Universidad Federal Fluminense, Brasil.

(2) Laboratório de Nutrición Experimental, Departamento de Nutrición y Dietética, Facultad de Nutrición, Universidad Federal Fluminense, Brasil.

\begin{abstract}
The protein quality of a flaxseed diet was evaluated by biological methods. Forty-eight recently weaned male Wistar rats with 21 days of life and average weight of $42 \mathrm{~g}$ were randomly divided in 4 groups $(n=12)$ and were fed, respectively, with a diet based on flaxseed added with casein (flaxseed group), casein alone (control group), casein with bigger concentration of oil and fibers to be compared to the flaxseed diet (modified control group) and a diet free of protein (non protein group). Animal weight, food and protein consumption were registered every other day to obtain the values for the Protein Efficiency Ratio (PER), Food Efficiency Ratio and Net Protein Ratio (NPR). At the end of the experiment, the nutritional value of the flaxseed diet was inside the reference values for protein quality; however, it was not equivalent to the casein diet.
\end{abstract}

Key words: Flaxseed, functional food, protein quality, growth, rats.

Este trabajo fue recibido el 18 de Julio de 2008 y aceptado para ser publicado el 10 de Noviembre de 2008

\section{INTRODUCCIÓN}

La semilla de linaza (Linum usitatissimum) tiene una larga historia de consumo en Europa y Asia. En Brasil, esta oleaginosa está siendo adicionada a panes, cereales para el desayuno, barras energéticas y otros productos de panificación. La linaza presenta algunas variedades de semillas que van del marrón oscuro al amarillo; en Brasil, la forma más común es la marrón, siendo más accesible que la dorada encontrada en Europa (1).

En las últimas décadas ha surgido un gran interés de la industria y los consumidores para los alimentos o los componentes alimenticios fisiológicamente activos, como la semilla de linaza, para promover beneficios a la salud, siendo llamados alimentos funcionales (2). Esta oleaginosa (tabla 1) posee un contenido en alto grado del ácido graso poliinsaturado alfa-linoléico (Omega-3), que representa en su composición 50 - 55\% de los ácidos grasos totales, y las fibras representan cerca de $40 \%$ de su peso total, siendo el $10 \%$ soluble y el $30 \%$ insoluble, además de las proteínas, ligninas, vitaminas y minerales (3). Tales sustancias se relacionan al potencial efecto beneficioso, como reducción en el riesgo del desarrollo de las enfermedades cardiovasculares, cáncer $(4,5)$, actividad anti-inflamatoria, efecto laxante y antioxidante, además de la prevención de síntomas de la menopausia (6).

El contenido de Omega-3 en la linaza es mayor que en cualquier otra semilla oleaginosa (4). Además de la presencia de este ácido, esta semilla también es rica en proteínas, como demostrado en algunos estudios que señalan que la composición aminoacídica encontrada en la proteína de la linaza es similar a la de la soja, considerada como una de la más nutritivas entre las proteínas de origen vegetal; albúmina y globulina representan cerca de $20 \%$ a $42 \%$ de la proteína de la linaza $(7,8)$.

Respecto a las fibras de esta oleaginosa, las insolubles aumentan el volumen de las heces fecales y reducen el tiempo del tránsito intestinal; y las solubles ejercen 
efecto hipoglicemiante e hipocolesterolémico, con la formación de un gel intra-luminal que disminuya la superficie de contacto con las vellosidades, reduciendo la absorción de colesterol y glucosa $(1,9,10)$. Con relación a las ligninas, el contenido de éstas en la semilla de linaza es 800 veces superior al de otros 66 alimentos vegetales evaluados (4).

Las fuentes proteicas alternativas están siendo bastante estudiadas, principalmente las de origen vegetal, siendo proteína de bajo costo y, por lo tanto, accesible a todos (7). Mientras que muchos grupos de la población prefieren consumir dietas ricas en proteína animal, el alto costo y la disponibilidad limitada de estas proteínas obligan a la mayoría de las naciones del tercer mundo al uso de proteína vegetal en detrimento de la de origen animal, convirtiéndose la soja y, más recientemente, la linaza en un potencial substituto de estas proteínas en los alimentos $(11,12)$. La oferta escasa de alimentos ricos en proteínas es una de las principales causas de desnutrición en los países en vías de desarrollo. No obstante, es posible aumentar la producción de alimentos proteicos procesados de bajo costo y de buena calidad para la alimentación humana, con el uso de la linaza, pues es una alternativa accesible para la industria y el mercado consumidor debido principalmente a su bajo costo $(13,14)$.

Considerando lo expuesto, este estudio tiene por objetivo evaluar la calidad proteica de una dieta a base de linaza, mediante métodos biológicos, en un experimento de 28 días.

\section{SUJETOS Y MÉTODOS}

\section{Animales}

Se utilizaron 48 ratas machos Rattus norvegicus, Albinus, Wistar, de 21 días de edad, recién destetados, con peso promedio de $42 \mathrm{~g}$. Éstos fueron mantenidos en el vivero en jaulas individuales de polipropileno, con temperatura constante $\left(22 \pm 2^{\circ} \mathrm{C}\right)$ e iluminación controlada, ciclo claro-oscuro $12 / 12 \mathrm{~h}$, recibiendo agua ad libitum durante todo el experimento. El protocolo de trabajo fue aprobado por el Comitê de Ética em Pesquisa - Hospital Universitario Antônio Pedro - Universidade Federal Fluminense, RJ, Brasil (parecer no 188/06) y todos los procedimientos siguieron las normas internacionales y del Colégio Brasileiro de Experimentação Animal (COBEA) para el cuidado y uso de animales de laboratorio.

\section{Grupos experimentales}

Los animales fueron divididos en 4 grupos $(n=12)$ : Linaza (GL), alimentado con dieta de linaza adicionada de 5,43\% de caseína; Control (GC), alimentado con dieta de caseína; Control Modificado (GCM), alimentado con dieta de caseína, teniendo una mayor concentración de aceite y fibras para equipararse a la dieta de linaza; Aproteico (GA) alimentado con dieta libre de proteína, que fue utilizado para la evaluación por el método biológico de Razón Proteica Neta (NPR).

El grupo GCM fue sometido a pareado alimentar (pair feeding) con GL, siendo ofrecida la misma cantidad de alimento consumido por este último y proporcionando así macronutrientes de forma equivalente.

\section{Dietas experimentales}

Todas las dietas eran isoproteicas con $10 \%$ de proteína en su composición e isocalóricas, siendo preparadas y balanceadas de acuerdo con las recomendaciones del American Institute of Nutrition - AIN-93 (15), según lo presentado en las tablas 2 y 3 .

\section{Recolección de datos}

El consumo diario fue determinado por medio de la diferencia de peso entre lo ofrecido y las sobras. La ingestión calórica total diaria fue estimada mediante el cálculo del valor calórico de los nutrientes, con base en el conocimiento de la composición y el consumo de las dietas usadas. Cada 2 días, durante todo el experimento, los animales fueron pesados en balanza digital con precisión de $0,5 \mathrm{~g}$.

\section{Evaluación biológica}

Para la evaluación biológica de la calidad proteica de las raciones se determinó la Razón de Eficiencia Proteica (PER). Este método tiene por base la variación de peso corporal en función de la cantidad de proteína ingerida. Considerando que ocurren variaciones en el total de pro-

\section{TABLA 1}

\section{Composición centesimal de la semilla de linaza}

\begin{tabular}{cccccc} 
Proteína & Lípidos & Carbohidratos & Fibras & Cenizas & Humedad \\
\hline 23,46 & 34,87 & 1,61 & 30,64 & 3,32 & 6,10
\end{tabular}


teína ingerida debido a las diferencias de calidad proteica de la dieta, es común medir la variación del peso como consecuencia global del funcionamiento de la proteína ingerida. PER es definido por la razón entre el aumento de peso de los animales y el consumo de proteína entre los días cero y $28^{\circ}(16)$.

El Coeficiente de Eficiencia Alimentaria (CEA) determina cuánto aumento de peso corporal promueve un gramo de alimento injerido y corresponde a la razón entre la variación del peso de los animales y la cantidad de alimento consumido. Para el cálculo del CEA fue con- siderada la variación de peso de los animales que ocurrió entre los días cero y $28^{\circ}$ y el consumo acumulativo de cada dieta experimental hasta el día $28^{\circ}(16)$.

La Razón Proteica Neta (NPR) consiste en sumar al aumento de peso del grupo de prueba, que recibió la dieta proteica, la pérdida de peso de un grupo equivalente que recibió dieta libre de proteína (16).

\section{Análisis estadístico}

El análisis de los resultados se realizó utilizando el programa estadístico Statgraphics Plus 6.0 aplican-

\section{TABLA 2}

\section{Composición de las dietas experimentales con $10 \%$ de proteína}

\begin{tabular}{lccc|}
\hline Componentes $(\mathbf{g} / \mathbf{1 0 0 g})$ & Linaza & Caseína & Caseína modificada \\
\hline caseína $^{1}$ & 5,43 & 11,80 & 11,80 \\
linaza $^{2}$ & 25,00 & ----- & ----- \\
almidón $^{3}$ & 54,10 & 61,20 & 58,20 \\
azúcar $^{4}$ & 10,00 & 10,00 & 10,00 \\
mix minerales $^{1}$ & 3,5 & 3,50 & 3,50 \\
mix vitaminas $^{1}$ & 1,00 & 1,00 & 1,00 \\
aceite $^{5}$ & ----- & 7,00 & 10,00 \\
celulosa $^{6}$ & ----- & 5,00 & 11,33 \\
b-colina & 0,25 & 0,25 & 0,25 \\
cistina $^{1}$ & 0,30 & 0,30 & 0,30 \\
total & $\mathbf{1 0 0 , 0 0}$ & $\mathbf{1 0 0 , 0 0}$ & $\mathbf{1 0 0 , 0 0}$
\end{tabular}

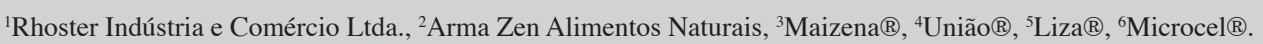

\section{TABLA 3}

Composición química centesimal de las dietas experimentales con $10 \%$ de proteína

\begin{tabular}{lccc}
\hline Composición química (g/100g) & Linaza & Caseína & Caseína modificada \\
\hline humedad (\%) & $1,95 \pm 0,08$ & $2,23 \pm 0,04$ & $2,35 \pm 0,03$ \\
lípidos (\% & $11,70 \pm 0,12$ & $7,79 \pm 0,30$ & $11,79 \pm 0,12$ \\
cenizas (\%) & $4,42 \pm 0,07$ & $4,27 \pm 0,02$ & $4,47 \pm 0,07$ \\
proteína (\%) & $10,18 \pm 1,06$ & $9,77 \pm 0,45$ & $9,75 \pm 0,45$ \\
fibras (\%) & $6,98 \pm 0,47$ & $5,00 \pm 0,34$ & $6,70 \pm 0,52$ \\
carbohidratos (\%) & 64,77 & 70,91 & 64,92 \\
VET (Kcal) & 405,10 & 392,83 & 404,79 \\
\hline VET = valor energético total. & & &
\end{tabular}


do análisis de varianza ANOVA de una vía, seguido por múltiples comparaciones mediante las pruebas de Scheffé y Bonferroni.

\section{RESULTADOS}

Según lo demostrado en la tabla 4, al final de los 28 días, el mayor consumo de alimento ocurrió en GC siendo significativamente superior a los otros dos grupos $(\mathrm{p}<0,0001)$; sin embargo GL presentó un valor estadístico superior a GCM. Lo mismo sucedió con el consumo de proteína, ya que GC presentó el mayor valor ( $\mathrm{p}<0,0001)$ y GL apenas demostró superioridad estadística cuando comparado a GCM.

La evolución ponderal se puede visualizar en la figura 1. De acuerdo con el peso inicial y final para el análisis del beneficio ponderal de los animales se observa que GL presentó mayor aumento de peso corporal cuando comparado a GCM y menor variación ponderal con significación estadística $(\mathrm{p}<0,0001)$ cuando comparado a GC (tabla 4).

\section{TABLA 4}

\section{Consumo total de dieta, proteína total y variación de peso de los animales al final del experimento}

\begin{tabular}{lccc}
\hline Grupos & $\begin{array}{c}\text { Consumo de dieta } \\
(\mathbf{g})\end{array}$ & $\begin{array}{c}\text { Consumo de proteína } \\
(\mathbf{g})\end{array}$ & $\begin{array}{c}\text { Variación de peso } \\
(\mathbf{g})\end{array}$ \\
\hline $\mathrm{GL}$ & $333,86 \pm 4,89 \mathrm{~b}$ & $34,38 \pm 0,50 \mathrm{~b}$ & $100,50 \pm 2,20 \mathrm{~b}$ \\
$\mathrm{GC}$ & $450,47 \pm 6,30 \mathrm{c}$ & $46,41 \pm 0,64 \mathrm{c}$ & $174,58 \pm 3,88 \mathrm{c}$ \\
$\mathrm{GCM}$ & $276,75 \pm 6,12 \mathrm{a}$ & $28,50 \pm 0,63 \mathrm{a}$ & $86,83 \pm 2,79 \mathrm{a}$ \\
\hline
\end{tabular}

\section{FIGURA 1}

\section{Evolución ponderal de los animales durante los 28 días de experimento}

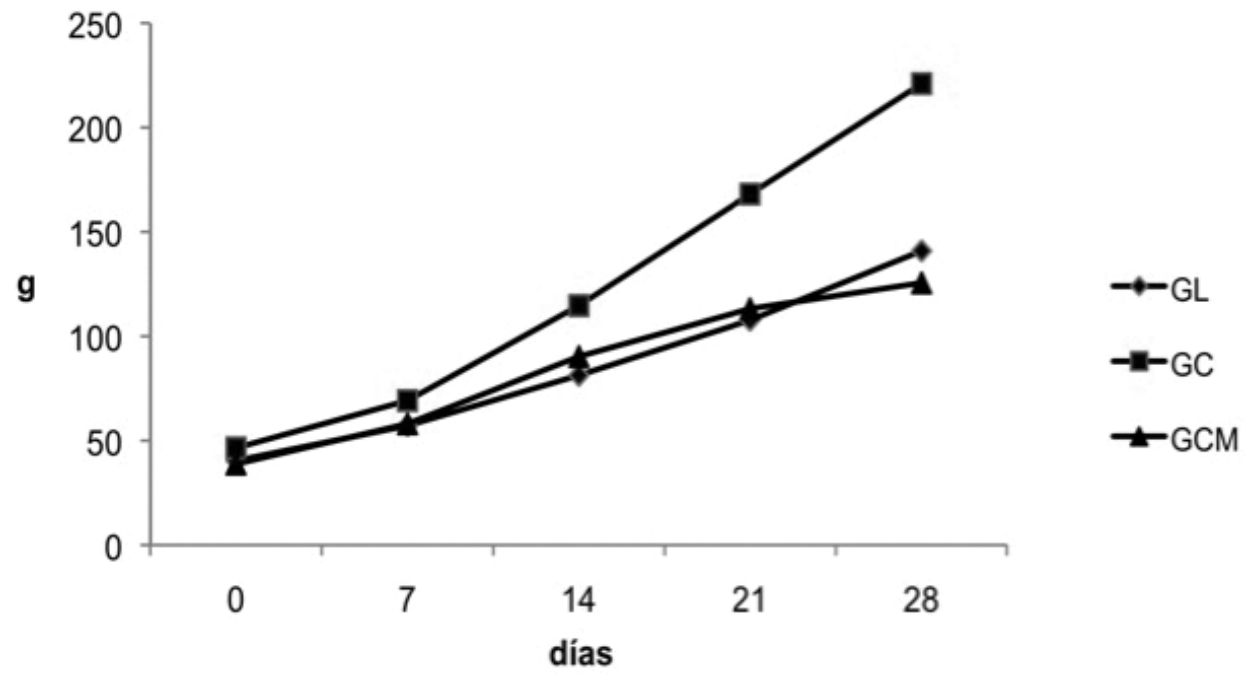


La evaluación de PER (figura 2) y de CEA (figura 3) demostró que las eficiencias proteica y alimentaria fueron similares en GL y GCM, siendo ambas significativamente inferiores cuando se compararon a GC $(\mathrm{p}<0,0001)$.

Respecto a los valores de NPR (figura 4), que constituyen una modificación del PER, el grupo que se alimentó con dieta a base de linaza (GL) presentó valo-

\section{FIGURA 2}

Razón de Eficiencia Proteica (PER) de los animales al final de los 28 días de experimento

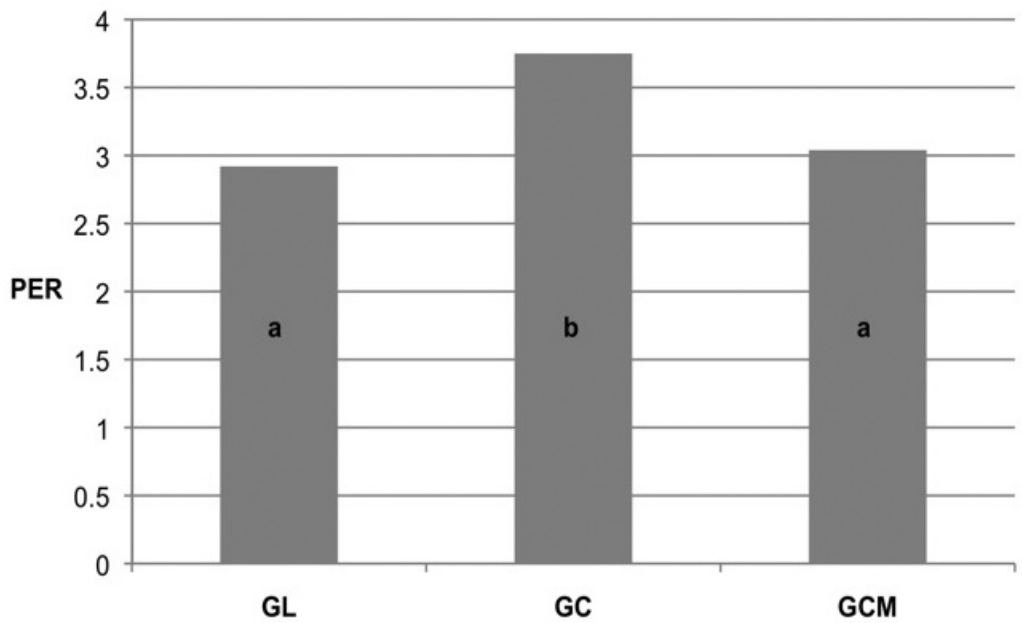

GL grupo linaza, GC grupo caseína, GCM grupo caseína modificada. Los valores representan el promedio de 6 ratas por grupo. Letras diferentes indican diferencia significativa $(\mathrm{p}<0,05)$. Prueba estadística ANOVA Bonferroni.

\section{FIGURA 3}

Coeficiente de Eficiencia Alimentaria (CEA) de los animales al final de los 28 días de experimento

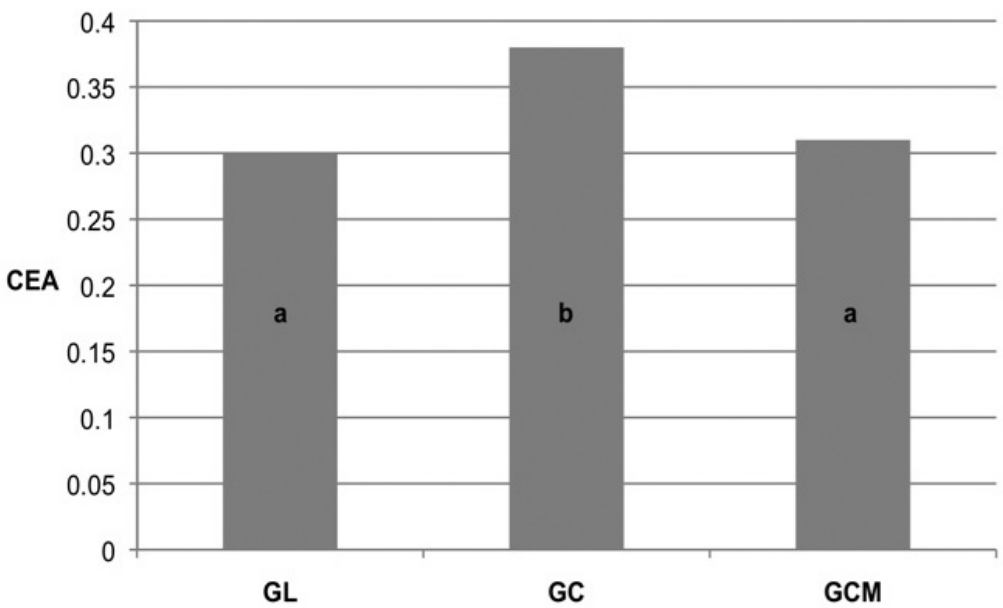

GL grupo linaza, GC grupo caseína, GCM grupo caseína modificada. Los valores representan el promedio de 6 ratas por grupo. Letras diferentes indican diferencia significativa $(\mathrm{p}<0,05)$. Prueba estadística ANOVA Bonferroni. 
res estadísticamente inferiores $(\mathrm{p}<0,001)$ a los grupos alimentados con dieta a base de caseína (GC) y caseína modificada (GCM).

\section{DISCUSIÓN}

Durante el crecimiento, además de las necesidades proteicas para la manutención del organismo, existen las exigencias para la formación de nuevos tejidos. Para garantizar que eso ocurra, las necesidades de energía del organismo también deben ser satisfechas, ya que habiendo deficiencias el organismo desvía las proteínas para producir energía. En los períodos del crecimiento intenso aumenta la necesidad proteica a ser ingerida, debido al aumento de las tasas de síntesis y degradación. Así, la calidad proteica llega a ser relacionada a su capacidad de satisfacer las necesidades del organismo, para promover adecuado crecimiento y manutención (17).

El consumo de dieta a base de linaza (GL) fue superior al consumo observado en GCM, y ambos fueron inferiores a GC; esto refleja un mayor consumo proteico en GL cuando se compararon a GCM y un menor consumo proteico cuando comparado a GC. Esto puede deberse a la cantidad de fibras y grasas presentes en las dietas ofrecidas a GL y GCM. Las fibras - solubles e insolubles - están presentes en gran cantidad en la semilla de linaza (28\%), haciendo con que los animales tengan una sensación más grande de saciedad, lo que conduce a menor consumo (9). Otro factor que puede haber contribuido para esto es la gran concentración de lipídos existentes en esta semilla. La cantidad de calorías en 100 gramos de linaza es de $396 \mathrm{kcal}$, siendo $109 \mathrm{kcal}$ de proteína y $287 \mathrm{kcal}$ de lipídos. Esto corresponde a 30,9g de lipídos y $24,4 \mathrm{~g}$ de las proteínas (8).

El menor consumo proteico de GCM en relación a GC sucedió porque ese grupo estaba pareado - pair feeding - con GL, recibiendo la misma oferta de cantidad de alimento. Así mismo GL presentó un consumo mayor, lo que se puede atribuir al gran porcentaje de fibras insolubles en la dieta a base de linaza, que aumentan el volumen de las heces fecales y reducen el tiempo de tránsito intestinal, haciendo que los animales vuelvan a consumir alimento más rápidamente (4).

Respecto al peso corporal, hubo homogeneidad entre GL y GCM, que fueron inferiores a GC. Esta observación se repite durante casi todo el experimento, puesto que solamente al día $28^{\circ}$ aparecen diferencias entre los tres grupos, cuando GL se presenta superior a GCM. Ratas Wistar alimentadas com dieta comercial (18) presentan peso corporal inicial de 49,50 $\pm 5,11 \mathrm{~g}$ y peso final de $147,52 \mathrm{~g} \pm 22,72$, los cuales se aproximan a los encontrados en este experimento en GL y GCM.

Como el consumo de alimento y de proteínas de GL fue mayor que el de GCM, el primero también presentó una variación ponderal más grande, en comparación con el segundo, lo que ya era esperado, pues más allá de la calidad, la cantidad de proteína ingerida interviene

\section{FIGURA 4}

\section{Razón Proteica Neta (NPR) de los animales al final de los 28 días de experimento}

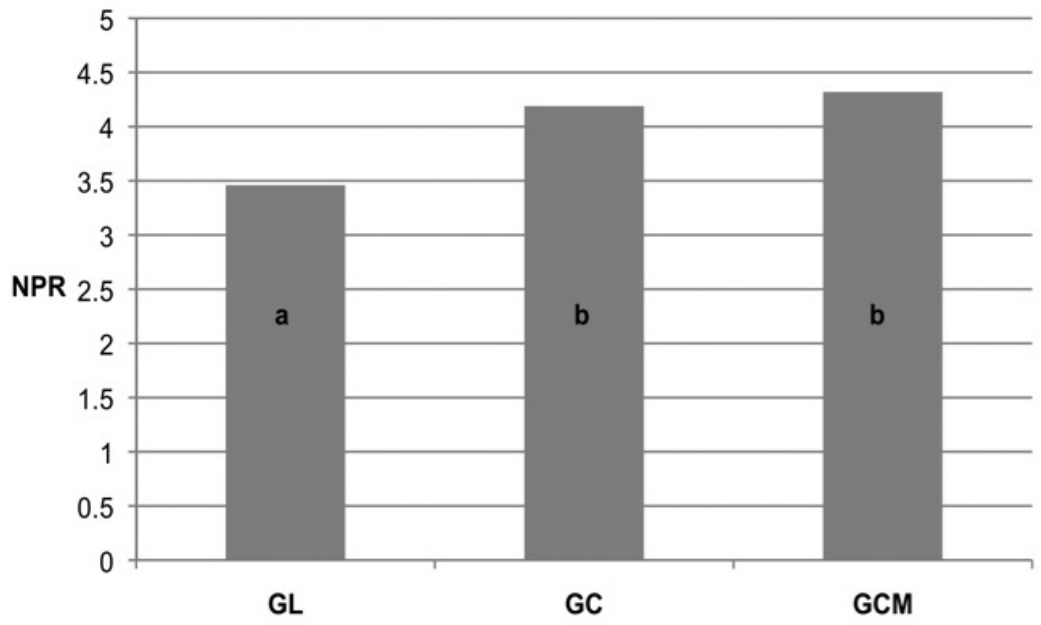

GL grupo linaza, GC grupo caseína, GCM grupo caseína modificada. Los valores representan el promedio de 6 ratas por grupo. Letras diferentes indican diferencia significativa $(\mathrm{p}<0,05)$. Prueba estadística ANOVA Bonferroni. 
directamente con un mayor crecimiento corporal, acompañado de una variación ponderal más grande (19). Esto se puede observar claramente, cuando comparamos GL y GCM con GC. Durante todo el experimento, este último presentó los mayores valores en cuánto al consumo de alimento y de proteínas y una variación ponderal más grande, que se atribuye no sólo a la mayor ingestión de alimento, mas también al alto valor biológico de la proteína presente en esa dieta.

El análisis de una dieta con mezcla de proteínas de origen vegetal y animal comparándola con una dieta a base de caseína encontró el mayor aumento de peso en los animales que habían recibido dieta con la proteína exclusivamente animal (20), de forma semejante a lo encontrado en este estudio. Sin embargo, resulta curioso que GL presentó una variación ponderal más grande que GCM, puesto que en la dieta ofrecida a este grupo la caseína también estaba presente como fuente proteica. Esto se puede atribuir al menor consumo de alimento y de proteínas en GCM. Otro posible factor que contribuye sería la presencia en una cantidad mayor de fibras insolubles en la dieta de GL, que actuarían aumentando el volumen de las heces fecales, reduciendo el tiempo del tránsito en el intestino grueso, lo que hace la eliminación fecal más fácil y más rápida (21); esto haría que los animales volvieran a consumir alimento en poco espacio de tiempo, pero podría no haber absorción adecuada de las proteínas ofrecidas.

La mayoría de las proteínas del origen animal tiene buena digestibilidad, lo que implica en una absorción eficiente de aminoácidos $(22,23)$. Las proteínas de origen vegetal son generalmente inferiores en su calidad proteica; esto justifica los menores valores de índices biológicos encontrados en GL en comparación con GC. Las variaciones en la digestibilidad pueden deberse a diferencias intrínsecas a la naturaleza de las proteínas y a la presencia de factores dietéticos (fibras, taninos y otras), que modifican la digestión y las reacciones químicas que liberan los aminoácidos y proteínas por procesos enzimáticos (24).

El método PER se utiliza como forma de evaluación del crecimiento y del beneficio de la proteína. El valor de PER arriba de 2 se relaciona con proteína de alta y buena calidad biológica y el valor de PER abajo de 1,5 con la proteína de baja y mala calidad (25). Siendo así, se puede afirmar que la dieta ofrecida a GL era de buena calidad, puesto que este grupo presentó PER igual a 2,9. Sin embargo, este resultado no se puede atribuir exclusivamente a la presencia de linaza en esta dieta, pues contenía también un pequeño porcentaje de caseína y estudios anteriores (26), con linaza como fuente exclusiva de proteína, la clasificaron como de baja calidad proteica, ya que presentó PER inferior a 1,5. La igualdad estadística encontrada en los valores de PER de GL y GCM se puede explicar por el hecho de estar en alimentación pareada, lo qué permitió evaluar el déficit del crecimiento relacionado con la calidad proteica de la dieta, y no con el menor aumento ponderal provocado por el bajo consumo de la misma, identificada en los experimentos anteriores (7). Durante todo el experimento GL presentó consumo de proteína equivalente a GCM, sin embargo mostró mayor variación ponderal, en comparación con este grupo.

En cuánto al CEA, el menor valor presentado por GL se puede explicar no sólo por la baja calidad proteica de la linaza, sino también por su alto contenido de fibras y de factores anti-nutricionales. El grupo GCM presentó valor de CEA numéricamente superior a GL, lo que se debe a la composición de su dieta, solamente con proteína de origen animal. No hubo diferencia estadística entre ellos, lo que puede ser justificado por la misma concentración de fibras en las dietas ofrecidas a ambos. Los grupos GL y GCM, cuando se compàraron a GC, presentaron resultado de CEA inferior.

Algunos factores de la dieta tienen papel importante al modificar el uso de la proteína, como las fibras y los fitatos, que pueden afectar la bio-utilización de diversos alimentos. Las fibras, además de aumentar las heces fecales y el flujo intestinal, pueden interactuar con la proteína haciendo difícil su absorción debido a la reducción de la digestión enzimática. Otra posibilidad de acción se relaciona con la viscosidad presente en la linaza durante el tránsito gastrointestinal, lo que favorece la formación de un gel coloidal, haciendo que la proteína tenga poco contacto con la mucosa intestinal (27).

Al analizar la influencia de la adición de un suplemento alimenticio alternativo - asociación de salvados, semillas vegetales y leche en polvo - en el crecimiento y el desarrollo de ratas (28), se encontró resultado similar al de este trabajo. También fue verificada la reducción del CEA con la dieta suplementada cuando se comparó a la dieta de caseína. Tal hecho se puede justificar por la gran cantidad de factores anti-nutricionales presentes en las semillas y los salvados que hacían parte del suplemento, los cuales inhibieron la absorción proteica (29), así como lo observado en la dieta a base de linaza.

Al determinarse los valores de NPR, se verificó que GL no era capaz de igualar los grupos alimentados con caseína. En otro estudio (7), el valor de NPR para el grupo alimentado con linaza como fuente exclusiva de la proteína fue inferior $(1,90 \pm 0,23)$ al de este experimento $(3,46 \pm 0,18)$. Debe ser considerado que en el actual experimento la dieta ofrecida a GL además de la linaza contenía un pequeño porcentaje de caseína. 
Resultado similar al de este estudio, con valor de 4,54 para NPR, se obtuvo trabajando con una dieta a base de leche en polvo, extracto soluble de soja y clara de huevo deshidratada (30).

Comparando los valores de PER y CEA encontrados en este estudio se observa una gran semejanza de comportamiento entre los grupos, pues en estos dos métodos de evaluación GL y GCM presentaron valores similares, pero estadísticamente inferiores a GC. Esto viene a confirmar lo existente en la literatura, pues una ración con buena calidad proteica tiende a promover un mayor peso corporal. Los valores encontrados para NPR se diferencian de lo descrito, puesto que este método no sólo evalúa la capacidad de la proteína de promover el aumento ponderal, sino también de mantener este aumento. Por esta razón, el grupo GL presentó valores inferiores a los demás grupos, debido a la proteína de bajo valor biológico contenida en esa dieta.

La dieta a base de linaza, aunque de buen provecho en función de la adición de caseína, no promovió crecimiento y mantenimiento adecuados del peso corporal en comparación con las otras dietas, puesto que de una forma general, GL presentó los menores valores referentes a todos los indicadores biológicos analizados. La dieta a base de caseína modificada - con fibras y aceite - presentó resultados similares a GL en algunos parámetros, puesto que la composición química de GCM fue especialmente equilibrada de acuerdo con la de la linaza, para así tener resultados más fidedignos.

Al final de este experimento se llegó a la conclusión de que el contenido de nutrientes de la dieta a base de linaza, a pesar de estar dentro del rango de referencia de calidad de proteínas, no era equivalente a la dieta a base de caseína, pues presentó valores inferiores a los demás grupos, en todos los indicadores biológicos analizados, lo que indica que no puede ser utilizada como una fuente de proteínas en los alimentos por sí sola, a pesar de sus beneficios para la salud.

\section{RESUMEN}

La calidad proteica de una dieta a base de linaza fue evaluada por medio de métodos biológicos. Fueron usados 48 Rattus norvegicus, Wistar, machos, con 21 días de vida, recién destetadas y con peso promedio de $42 \mathrm{~g}$. Los animales fueron divididos en 4 grupos $(n=12)$ que recibieron, respectivamente, dieta a base de linaza adicionada de caseína, caseína, caseína con mayor concentración de aceite y fibras para equipararse a la dieta de linaza y dieta libre de proteína. Cada dos días fueron registrados el peso animal, el consumo de alimento y de proteína para aplicación de los métodos de evaluación biológica Razón de Eficiencia Proteica
(PER), Coeficiente de Eficiencia Alimentaria (CEA) y Razón Proteica Neta (NPR). Al final del experimento, el valor nutricional de la dieta de linaza estuvo dentro de los valores de referencia de calidad proteica, pero no fue equivalente al de la dieta a base de caseína.

Palabras claves: Linaza, alimento funcional, calidad proteica, crecimiento, ratas.

Dirigir la correspondencia a:

Srta.

Kátia Calvi Lenzi de Almeida

Fisioterapeuta

CREFITO: 95912-F

Doutoranda em Patologia /UFF

Laboratório de Nutrição Experimental

(21) 26299860 / (22) 92558597 (cel)

E-mail: calvilenzi@gmail.com

\section{BIBLIOGRAFÍA}

1. WHO and FAO joint consultation: fats and oils in human nutrition, Nutr Res 1995; 38:202-205.

2. Position of the American Dietetic Association: Phytochemicals and functional foods, J Am Diet Assoc 1995; 95:493-496.

3. Calder PC. Immunoregulatory and anti-inflammatory effects of n-3 polyunsatured fatty acids. Braz J Med Biol Res 1998; 31:467-490.

4. Thompson LU, Robb P, Serraino M, Cheung F. Mammalian lignan production from various foods. Nutr Cancer 1991; 16:43-52.

5. Hanf V, Gonder U. Nutrition and primary prevention of breast cancer: foods, nutrients and breast cancer risk. Eur J Obstet Gynecol Reprod Biol 2005; 123:139-149.

6. Caragay AB. Cancer-preventive foods and ingredients. Food Technol 1992; 46:65-68.

7. Gómez MEDB. Modulação da composição de ácidos graxos poliinsaturados ômega-3 de ovos e tecidos de galinhas poedeiras, através da dieta. I. Estabilidade oxidativa [tese]. São Paulo (SP): Universidade de São Paulo; 2003.

8. Turatti JM. A importância dos ovos numa dieta saudável. Óleos e grãos 2001; 9:22-24.

9. Carter JF. Potential of flaxseed and flaxseed oil in baked goods and other products in human nutrition. Cereal Foods World 1993; 38:753-759.

10. Leaf A, Weber PC. Cardiovascular effects of n-3 fatty acids. N Engl J Med 1988; 318:549-557.

11. Souza G, Valle JLE, Moreno I. Efeitos dos componentes da soja e seus derivados na alimentação humana. Bolet Soc Bras Ciên Tecnol Alim 2000; 34:61-69. 
12. Roe DA. Effects of drugs on vitamin needs. Ann N York Acad Sci 1992; 669:156-163.

13. Pszczola DE. Ingredients that shape the new millennium. Food Technol 1999; 53:138-142.

14. Riley PA. Free radicals in biology: oxidative stress and the effect of ionizing radiation. Int J Radiat Biol 1994; 65:27-33.

15. Reeves PG, Nielsen FH, Fahey GC Jr. AIN-93 Purified diets for laboratory rodents: final report of the American Institute of. Nutrition ad hoc writing committee on the reformulation of the AIN-76A rodent diet. J Nutr 1993; 123:1939-1951.

16. Campbell JA. Method for determination of PER \& NPR. In: Food and Nutrition Board. Committee on Protein Quality. Evaluation of protein quality. Washington,D.C., NAS/NRC, 1963, pp. 31-32.

17. Lajolo FM, Tirapegui J. Proteínas e Aminoácidos. In: Dutra de Oliveira JE. Marchini JS. Ciências Nutricionais. São Paulo, Sarvier, 1998, pp. 41-65.

18. Guerra MO, Peters VM. Morfometria de ratos Wistar: peso e medidas corporais. Rev Ciênc Bioméd 1995; 15:65-74.

19. Ward WE, Jiang FO, Thompson LU. Exposure to flaxseed or purified lignan during lactation influences rat mammary gland structures. Nutr Cancer 2000; 37:187-192.

20. Monteiro JBR, Costa NMB, Esteves EA, Milagres KH. Avaliação da qualidade protéica de dois formulados em pó à base de soja, enriquecidos com zinco, selênio e magnésio para utilização em nutrição enteral. Ciênc Tecnol Aliment 2004; 24:6-10.

21. Mattos LL, Martins IS. Consumo de fibras alimen- tares em população adulta. Rev Saúde Pública 2000; 34:50-55.

22. Tagle MA. Nutrição. São Paulo, Artes Médicas, 1981.

23. Bressani R. Revisión sobre la calidad del grano de frijol. Arch Latinoam Nutr 1989; 39:419-442.

24. Informe de una Reunión consultiva conjunta FAO/ OMS/UNU de expertos. Necesidades de energía y de proteínas. Ginebra, OMS, 1985.

25. Friedman M. Nutritional value of proteins from different food sources, a review. J Agric Food Chem 1996; 44:6-29.

26. Morais AAC, Silva AL. Valor nutritivo e funcional da soja. Rev Bras Nutr Clín 2000; 15:306-315.

27. Frias AC, Sgarbieri VC. Guar gum effects on food intake, blood serum lipids and glucose concentrations of Wistar rats. Plant Foods Hum Nutr 1998; 53:15-28.

28. Pacheco JT, Daleprane JB, Boaventura GT. O efeito da alimentação alternativa nos indicadores biológicos e químicos de ratos em crescimento alimentados com a dieta do município de Quissamã/RJ. Rev Saúde Com 2007; 3:35-47.

29. Pita MCG, Piber NE, Carvalho PR, Mendonça JCX. Efeito da suplementação de linhaça, óleo de canola e vitamina E na dieta sobre as concentrações de ácidos graxos poliinsaturados em ovos de galinha. Arq Bras Med Vet Zootec 2006; 58:925-931.

30. Arhontaki J. Desenvolvimento e avaliação de formulações para alimentação de idosos [dissertação]. Viçosa (MG): Universidade Federal de Viçosa, 1990. 